\title{
Réappropriation du patrimoine autochtone : défis et nouvelles pratiques muséales et archivistiques
}

\author{
Melissa Vernier \\ Professeure d'histoire, programme en étude des enjeux humains et sociaux \\ Université de Hearst
}

\section{Mots clés}

Patrimoine culturel autochtone, rapatriement, pratiques muséales, pratiques archivistiques, Commission de vérité et réconciliation, décolonisation

\section{Résumé}

Le rapport de la Commission de vérité et réconciliation du Canada (2015) fait le point sur la colonisation et les politiques du gouvernement fédéral ayant porté atteinte à l'identité culturelle des peuples autochtones du pays. Que cela ait été intentionnel ou non, les institutions de la mémoire - musées, centres d'archives et bibliothèques - ont contribué à cet héritage colonial par l'imposition d'un régime d'historicité de conservation et d'interprétation occidentale. Le mouvement pour la réappropriation du patrimoine culturel autochtone, mené tant par les groupes autochtones que par les institutions de la mémoire elles-mêmes, cherche à corriger cette situation par des mesures aussi variées que le rapatriement, la restitution, la gestion partagée et l'autogestion des collections autochtones ainsi que la création de partenariats et de projets collaboratifs. Cette étude analyse d'abord le contexte sociohistorique dans lequel évolue le mouvement pour la réappropriation du patrimoine culturel autochtone pour ensuite présenter les réformes et les pratiques exemplaires des institutions de la mémoire issues de ce mouvement. Ces réformes témoignent de deux tendances distinctes, soit des stratégies d'autonomisation ou de collaboration. L'étude conclut sur quelques recommandations quant aux mesures institutionnelles à privilégier pour les prochaines décennies.

\section{Introduction}

Usurpation de leur souveraineté politique et territoriale, adoption de lois fédérales contraignantes et discriminatoires à leur égard, atteinte à leur identité culturelle, paupérisation et dégénérescence de leurs communautés, voilà le contexte qui a façonné I'histoire des Premières Nations, des Métis et des Inuits (PNMI) du Canada depuis la colonisation. Les institutions occidentales de la mémoire - musées, centres d'archives et bibliothèques - ont contribué à cet héritage colonial en imposant leur régime d'historicité de conservation et d'interprétation scientifique à des peuples de tradition plutôt orale et de mémoire vivante. 
Faisant enfin rupture avec ce lourd passé colonialiste de soumission aux institutions occidentales, les PNMI revendiquent maintenant le droit à l'autodétermination et à l'autogestion de leurs communautés, droit qui s'étend à la problématique de l'interprétation, de la conservation et de la transmission de l'héritage culturel. Dans ce nouveau contexte, le rôle et les pratiques occidentales des musées, centres d'archives et bibliothèques sont donc remis en question. Longtemps tenus silencieux à l'égard de leur propre histoire et du traitement réservé à leurs biens culturels, les peuples autochtones exigent maintenant un droit de regard sur la gestion de leur patrimoine et de leurs savoirs traditionnels.

Cet article examine ce mouvement pour la réappropriation du patrimoine culturel et documentaire des communautés autochtones du Canada ainsi que les défis et les nouvelles pratiques muséales et archivistiques issus de cette problématique.

Afin d'atteindre cet objectif, nous retraçons d'abord l'historique du mouvement en décrivant les dispositions et recommandations internationales, nationales et législatives ayant mené à la reconnaissance du droit à l'autodétermination et au rapatriement de l'héritage culturel. Nous discutons ensuite de l'impact de ces dispositions sur les initiatives et les pratiques muséales et archivistiques mises en place au Canada pour l'amélioration de leurs relations institutionnelles avec les communautés autochtones. Enfin, nous proposons quelques pistes de réflexion sur les avantages et les désavantages de ces pratiques et sur le travail qu'il reste à faire pour mener à bien les activités futures de préservation et de mise en valeur des documents et des collections autochtones.

\section{Historique du mouvement pour la réappropriation du patrimoine des communautés autochtones}

Le mouvement pour la réappropriation du patrimoine culturel et documentaire des communautés autochtones du Canada s'inscrit dans un courant international de décolonisation et de reconnaissance des droits de l'homme (Morse, 2012). Ce contexte se développe au lendemain de la Deuxième Guerre mondiale, notamment avec la Déclaration universelle des droits de l'homme (DUDH) qui dénonce les actes de barbarie commis pendant la guerre et qui cherche à prévenir de tels traitements inhumains en reconnaissant que « [t]ous les êtres humains naissent libres et égaux en dignité et en droits » (Nations Unies, 1948, p. 72).

Au Canada, ce contexte de justice sociale de l'après-guerre donne naissance à divers groupes de pression autochtones comme le North American Indian Brotherhood (1945) et l'Assemblée des Premières Nations (APN) (1982) qui défendent les droits des PNMI à Ottawa (Dickason, 2006). À cela s'ajoute la Charte canadienne des droits et libertés enchâssée dans la constitution canadienne de 1982 qui réaffirme la reconnaissance des libertés fondamentales de la personne telles que formulées dans la DUDH et qui reconnaît en plus les droits « ancestraux, issus de traités ou autres - des peuples autochtones du Canada » (Gouvernement du Canada, 1982, art. 25). 
C'est dans ce climat d'organisation et d'affirmations qu'auront lieu les premières revendications autochtones en matière d'héritage culturel, notamment avec le boycottage organisé par la nation crie du Lac Lubicon en 1988 de l'exposition Le souffle de l'esprit - les traditions artistiques des premiers habitants du Canada, une présentation du musée Glenbow préparée à l'occasion des Jeux olympiques d'hiver de Calgary sans l'avis des peuples autochtones concernés (Soulier, 2015, p. 99).

Mobilisées par une telle démonstration de principe, les communautés autochtones, appuyées par l'APN, entreprennent une série de rencontres avec les musées pour « développer un système et des stratégies éthiques permettant aux peuples autochtones et aux institutions culturelles de travailler ensemble pour représenter l'histoire et la culture autochtones » (Association des musées canadiens \& Assemblée des Premières Nations, 1992, p. 1). Cette initiative débouche sur la création du Groupe de travail sur les musées et les Premières Nations - parrainé par l'Association des musées canadiens (AMC) et l'APN - qui publie en 1992 un rapport faisant état de ses recommandations. Parmi celles-ci, notons, entre autres : 1) l'établissement de partenariats équitables entre les PNMI et les musées canadiens qui reconnaissent et respectent les connaissances, les approches, les interprétations et les capacités de cogestion de chacun des partis intéressés ; 2) le droit d'accès physique des PNMI aux collections autochtones des musées ; 3 ) le droit des communautés autochtones de participer aux conseils exécutifs et administratifs des musées ; 4) le droit au rapatriement du patrimoine culturel autochtone incluant les restes humains, les objets sacrés ou encore les objets de la culture matérielle par restitution, réversion, transfert de titre, prêt de matériel, reproduction d'artefacts ou gestion partagée du matériel concerné ; 5) l'établissement de programmes de formation professionnelle et technique pour le personnel autochtone ainsi que l'établissement de programmes de sensibilisation à la culture et aux perspectives autochtones du personnel non autochtone; 6 ) la création d'un fonds spécial de financement pour la mise en œuvre des recommandations du rapport et l'établissement de centres culturels et musées autochtones (AMC \& APN, 1992, p. 8-12).

Concernant le droit au rapatriement de l'héritage culturel autochtone, le Groupe de travail sur les musées et les Premières Nations a pris connaissance de la loi américaine, votée en 1990, sur le rapatriement et la protection des sépultures autochtones (Native American Graves Protection and Repatriation Act), sans toutefois faire de recommandations pour un projet législatif semblable. Le rapport mentionne que « tous étaient d'avis que, pour le moment, il est préférable d'encourager les musées et les peuples autochtones à travailler en collaboration pour résoudre les problèmes reliés à la gestion, le soin et la garde des objets culturels » (AMC \& APN, 1992, p. 7). En conséquence, les institutions canadiennes de la mémoire ne sont pas soumises à l'appareil législatif national en matière de rapatriement comme c'est le cas aux ÉtatsUnis, mais plusieurs ont entrepris leurs propres démarches de négociations avec les peuples autochtones (Morse, 2012, p. 134).

C'est ainsi que, lorsque les Nisga'a négocient avec Ottawa et la Colombie-Britannique leurs droits ancestraux et territoriaux, l'entente conclue en 1999 prévoit des dispositions quant à la protection, la propriété ainsi que le droit à l'autogestion et au rapatriement de 
leur héritage culturel. Le traité va même jusqu'à décrire les prévisions que doivent prendre deux musées canadiens - le Musée canadien des civilisations (aujourd'hui le Musée canadien de l'histoire) et le Royal British Columbia Museum - pour l'établissement de conventions de garde et le retour d'artefacts Nisga'a (Government of Canada, 1999, chap. 17, paragr. 7-34, p. 224-228 ; Morse, 2012, p. 137).

Sur la scène internationale, l'Assemblée générale de l'ONU adopte, en 2007, la Déclaration sur les droits des peuples autochtones. Ce manifeste reconnaît le droit des peuples autochtones, entre autres, à la protection, à la conservation, au développement et à la transmission de leur histoire, de leur héritage culturel et de leurs savoirs traditionnels ainsi qu'au rapatriement de leurs restes humains et de leurs biens culturels et spirituels (Nations Unies, 2007, art. 11-13, 31, p. 12). Bien qu'il s'agisse d'une déclaration de principes et non d'une loi internationale, elle recommande aux États membres de prendre « les mesures appropriées, y compris législatives, pour atteindre les buts de la présente Déclaration » (Nations Unies, 2007, art. 38, p. 14). Ce faisant, la Déclaration donne lieu à l'opposition de quatre États réfractaires - le Canada, les ÉtatsUnis, l'Australie et la Nouvelle-Zélande - se prononçant principalement contre le principe des droits collectifs autochtones dans la mesure où ceux-ci pourraient éclipser les droits individuels des citoyens non autochtones (Edwards, 2007, A4). Plus récemment, en 2014, lorsque les Nations Unies organisent une nouvelle Conférence mondiale sur les droits des peuples autochtones, tenue à New York, le Canada est alors la seule nation à continuer à s'opposer à la nouvelle déclaration. Son objection s'articule autour de la notion « free, prior and informed consent, before adopting and implementing legislative or administrative measures that may affect them » (United Nations, 2014, art. 3), croyant que cette formulation pourrait être interprétée comme un droit de veto autochtone non conforme au droit canadien (Lum, 2014). À deux reprises donc, le Canada s'est dressé contre les déclarations de principes des Nations Unies en matière de droits autochtones, quoique sans nécessairement remettre en question les articles en matière d'autogestion et de réappropriation du patrimoine culturel.

Au pays, l'APN réagit mal à la position réfractaire du Canada de 2007, le chef national de l'époque Phil Fontaine déclarant « [i]t is disappointing to see this government vote against recognizing the basic rights of Canada's first peoples » (cité dans Lambert, 2007, A17). L'APN répond néanmoins à l'affront en publiant, la même année, une politique sur le droit inhérent des PNMI à l'autogestion de leurs données qui fait suite aux recommandations de la Déclaration des Nations Unies. Cette politique, intitulée Propriété, contrôle, accès et possession, tente de « décoloniser la recherche » et remet en question la gestion des données autochtones par les institutions fédérales, provinciales ou académiques non autochtones qui, dans le passé, ont abusé de leur pouvoir en adoptant des pratiques non respectueuses ni du droit intellectuel autochtone ni du droit humain (APN, 2007, p. 13). Elle recommande plutôt aux collectivités autochtones de jouer un plus grand rôle et de se charger elles-mêmes de la gestion de leurs renseignements depuis « la collecte à la transmission en passant par l'analyse » (APN, 2007, p. 5), et ce, selon le principe que l'information, les données et les analyses produites seraient ainsi plus fidèles au vécu, au contexte, à la philosophie et aux besoins autochtones. 
Suivant l'exemple des musées avec un peu de retard, les bibliothèques municipales, représentées par le Conseil des bibliothèques municipales, provinciales et territoriales (Provincial/Territorial Public Library Council - PTPLC), montent, en 2008, un groupe de travail (Aboriginal Library Services Working Group) pour examiner leurs relations avec les peuples autochtones. S'inspirant de la roue médicinale, le rapport du groupe de travail élabore un cadre conceptuel - « Library practice wheel » - permettant aux bibliothèques d'évaluer leurs services en fonction des besoins et des valeurs autochtones, et ce, en donnant des exemples de saines pratiques pour chacun des quatre quadrants de la roue : leadership, composition, participation et innovation (Cavanagh, 2009). Ce rapport conclut en émettant une série de recommandations pour chaque autorité (territoriale, provinciale, régionale et municipale). Ces recommandations prônent, entre autres, la mise en place de programmes de formation professionnelle et interculturelle, la collaboration avec les communautés autochtones, l'évaluation régulière des services autochtones ainsi que l'adoption des principes fondamentaux d'équité, d'intégration, de diversité, de flexibilité, de partage et d'autonomisation (Cavanagh, 2009).

C'est dans ce contexte de collaboration et de décolonisation de la recherche, du savoir et de la mémoire que s'inscrit la Commission de vérité et réconciliation du Canada. Chargée d'enquêter sur l'affaire des pensionnats autochtones, la Commission devait également proposer un processus de guérison et de réconciliation pour les survivants et leurs familles et créer un centre national de recherche à la mémoire des victimes (Truth and Reconciliation Commission, 2012, p. 1, 17-18). Pour remplir un tel mandat, la Commission a dû procéder à de nombreuses collectes de témoignages et de données autochtones, à des recherches en dépôts d'archives gouvernementales, paroissiales, universitaires et privées et à la numérisation de la documentation recueillie (TRC, 2012, p. 13).

Le rapport intermédiaire de la commission, publié en 2012, témoigne toutefois d'un refus flagrant de coopération de certains archivistes d'église et de la part du gouvernement canadien, en particulier de Bibliothèque et Archives Canada et du département des Affaires autochtones et Développement du Nord Canada, ces institutions ayant limité considérablement l'accès ou l'utilisation des bases de données et des documents pertinents à l'enquête des commissaires (TRC, 2012, p. 16). La Commission s'inquiète également des coûts de reproduction des 5 à 50 millions de dossiers estimés, qui pourraient excéder le budget de 60 millions de dollars dont elle dispose et compromettre la réalisation de son projet de Centre national de recherche (TRC, 2012, p. 16-17). Les recommandations du rapport de 2012 en matière de patrimoine culturel portent ainsi sur le besoin de contraindre le gouvernement canadien et les églises à produire tous les documents pertinents à leur enquête, et ce, en assumant la plus grande part des frais de production et de numérisation de la documentation (TRC, 2012, p. 17).

Venant à peine d'être publié, le rapport final de la Commission (2015) accuse carrément le gouvernement fédéral d'avoir perpétré un génocide culturel contre les PNMI. Parmi les 94 recommandations formulées, la Commission préconise une révision des politiques et pratiques muséales et archivistiques, critiquant particulièrement 
Bibliothèque et Archives Canada afin qu'elle se conforme aux principes de la Déclaration des Nations Unies sur les droits des peuples autochtones et qu'elle rende ses fonds et ressources documentaires portant sur les pensionnats autochtones accessibles au public (TRC, 2015, art. 69, p. 8). La Commission recommande, par ailleurs, au gouvernement fédéral de fournir des fonds à l'AMC et à l'Association des archivistes canadiens afin qu'elles puissent procéder à un examen national des relations institutionnelles des musées et des centres d'archives avec les peuples autochtones (TRC, 2015, art. 67, 70, p. 8). Des fonds fédéraux devraient également être alloués à la mise en œuvre de projets de recherche, de commémoration et de réconciliation organisés en collaboration avec les peuples autochtones (TRC, 2015, art. $68,72,79-83$, p. 8-9) et particulièrement pour la création d'un Centre de recherche national de vérité et réconciliation (TRC, 2015, art. 78, p. 9). Enfin, la Commission exhorte les centres d'archives à remettre une copie de tous les documents relatifs aux pensionnats autochtones au Centre de recherche national de vérité et réconciliation (TRC, 2015, art. 77, p. 9).

Devant ces conclusions et recommandations de la Commission, le gouvernement Harper a hésité à s'engager officiellement, rappelant plutôt les excuses déjà présentées aux peuples autochtones à l'égard des pensionnats et les démarches prises par le gouvernement canadien conservateur dans le passé pour améliorer ses relations avec les PNMI. Dans son allocution à la Chambre des communes du 2 juin 2015, le premier ministre a tout simplement déclaré que « [...] le gouvernement va examiner le rapport et les recommandations de la commission avant de déterminer les prochaines étapes » (Gouvernement du Canada, 2015).

Alors que d'autres nations, comme les États-Unis et la Nouvelle-Zélande ${ }^{1}$, ont adopté des lois et des stratégies institutionnelles forçant les musées, les centres d'archives et les bibliothèques à transformer leurs pratiques en fonction des principes établis par la Déclaration des Nations Unies, force est de constater que le gouvernement canadien a jusqu'à présent réagi plutôt lentement et prudemment aux recommandations des déclarations et commissions des dernières années. La position du nouveau gouvernement libéral de Justin Trudeau est toutefois plus encourageante. Sur la page officielle du parti, on peut lire :

Nous appuierons le travail de réconciliation et poursuivrons le processus nécessaire de découverte de la vérité et de guérison. Pour ce faire, nous collaborerons avec les provinces, les territoires, les Premières Nations, les Métis et les Inuits pour mettre en œuvre les recommandations de la Commission de vérité et réconciliation, à commencer par l'application de la Déclaration des Nations Unies sur les droits des peuples autochtones (Parti libéral du Canada, 2015).

\footnotetext{
${ }^{1}$ En Nouvelle-Zélande, les musées, bibliothèques et centres d'archives doivent adhérer aux principes du Traité de Waitangi qui crée une nation biculturelle, soit britannique et maorie. Ainsi, les institutions de la mémoire doivent présenter l'héritage double de la nation et la plupart sont administrées en collaboration avec les Maoris (Morse, 2012).
} 
Reste à voir si ces promesses électorales seront tenues et comment il interprétera les articles en matière d'autogestion et de réappropriation du patrimoine culturel.

\section{Stratégies, politiques et initiatives canadiennes pour la décolonisation des pratiques muséales et archivistiques}

Malgré le fait que le gouvernement canadien s'est entêté dans son opposition aux déclarations de principes de l'ONU, situation que dénonce d'ailleurs la Commission de vérité et réconciliation du Canada, le mouvement pour la réappropriation du patrimoine culturel autochtone amorcé par ces dispositions et recommandations a donné lieu à une multitude de stratégies, de politiques, d'initiatives et de pratiques muséales, archivistiques et numériques plus sensibles aux questions autochtones. Ces dernières prônent soit le rapatriement et l'autogestion des collections autochtones, soit la création de partenariats et de projets collaboratifs.

\section{Stratégies d'autonomisation}

Nous qualifions ici de «stratégies d'autonomisation », concept que nous empruntons d'ailleurs à Dubuc et Turgeon (2004), les initiatives muséales et archivistiques qui s'alignent sur les principes de la politique Propriété, contrôle, accès et possession (APN, 2007) des données autochtones. II s'agit donc de pratiques qui cherchent à décoloniser la recherche ${ }^{2}$ et la mémoire en redonnant aux peuples autochtones la propriété et le contrôle de leurs biens culturels. On compte parmi ces stratégies, les politiques de rapatriement et de restitution ainsi que les projets de musées et de centres culturels ou archivistiques autochtones indépendants.

\section{Politiques de rapatriement et de restitution}

Suivant l'exemple de la loi américaine sur le rapatriement et la protection des sépultures autochtones (NAGPRA, 1990), les recommandations du Groupe de travail sur les musées et les Premières Nations (1992) et les exigences du traité des Nisga'a, plusieurs musées canadiens entreprennent très tôt l'élaboration de politiques de rapatriement et de restitution de leurs collections autochtones. À cet égard, la Société du Musée canadien des civilisations mène le bal et passe plusieurs réformes internes avec, entre autres, sa Politique sur les ossements humains et sa Politique sur le rapatriement (Morse, 2012, p. 132-135). Selon cette dernière politique, le Comité des collections reçoit et étudie chaque demande officielle de rapatriement en tenant compte de quatre facteurs :

\footnotetext{
2 Pour une réflexion approfondie sur la décolonisation de la recherche et sur les méthodes de recherche et de collectes de données plus respectueuses des participants autochtones et de leur culture, le lecteur peut consulter l'étude de cas maori de Tuhiwai Smith (2008). Au Canada, outre le PCAP, plusieurs protocoles au sujet de l'éthique de la recherche avec les peuples autochtones privilégient la méthode de la recherche-action participative (Association des femmes autochtones du Canada, 2011 ; Femmes autochtones du Québec Inc., 2012 ; Association des Premières Nations du Québec et du Labrador, 2015).
} 
i) le lien historique du ou des demandeurs avec les restes humains ou les objets visés;

ii) les conditions dans lesquelles le matériel demandé a été acquis par le musée;

iii) la possibilité de demandes rivales relatives au matériel;

iv) le caractère des objets [...] (SMCC, 2001, art. 5.3, p. 4).

Lorsque le rapatriement est approuvé, la SMCC conserve cependant un droit de reproduction sur les documents et objets de la collection nationale rapatriés, et ce, à des fins de gestion et de recherche (SMCC, 2001, art. 6.4, 6.5, p. 5-6).

D'autres musées, comme le Royal Ontario Museum et le Royal British Columbia Museum, suivront le pas en adoptant leurs propres politiques de rapatriement (ROM, 2001, Repatriation of Canadian Aboriginal objects; ROM, 2002, Repatriation of human remains of the Aboriginal Peoples of Canada; RBCM, 2003, Aboriginal material operating policy). En Alberta, les musées - entre autres le Royal Alberta Museum et le Musée Glenbow - sont toutefois plutôt soumis à deux lois provinciales : First Nations Sacred Ceremonial Objects Repatriation Act (2000) et Blackfoot First Nations Sacred Ceremonial Objects Repatriation Regulation (2004).

Toutes ces politiques se ressemblent à plusieurs égards : par le rapatriement sur demande ou selon les exigences d'un traité ou d'une entente particulière avec une PNMI ; par l'examen de la recevabilité des demandes au cas par cas selon un certain nombre de critères préétablis ; et par l'examen des revendications potentielles avancées par des nations autochtones rivales. Certaines politiques prévoient également la possibilité de négocier avec les communautés autochtones requérantes des ententes de garde partagée, de prêts ou de solutions de rechange au rapatriement (par exemple, une entente de rapatriement virtuel par la numérisation des objets et des documents d'une collection).

La législation albertaine fait toutefois exception en matière de définition des objets admissibles pour restitution. Alors que la plupart des politiques de rapatriement des musées incluent les restes humains, les objets de sépulture ou sacrés ainsi que les objets culturels et ethnographiques définis dans son sens plutôt large, les politiques albertaines sont plutôt restrictives. Celles-ci se limitent à la restitution des objets sacrés qui continueront d'être utilisés activement selon leurs fonctions traditionnelles : « [a] society may make an application for the repatriation of a sacred ceremonial object only if the society has received a request from an individual who has agreed to put that sacred ceremonial object back into use as a sacred ceremonial object » (AR, 2004, art. 4.2 , p. 2). Selon cette interprétation, les objets culturels ou même les objets sacrés tombés en désuétude ne qualifient donc pas pour le rapatriement au peuple autochtone d'origine.

Le projet de restitution de l'Université de Victoria d'une collection de peintures d'enfants autochtones créées lors de leur séjour au pensionnat de Port Alberni se démarque 
toutefois de ces politiques muséales de rapatriement plus conventionnelles. Mené par la professeure d'anthropologie Andrea N. Walsh, le projet se distingue par l'initiative de rapatriement prise par l'Université de Victoria et non par une communauté autochtone, comme le veut la pratique muséale d'une demande officielle. Ayant reçu les précieuses peintures de la famille de Robert Aller (professeur d'art de l'école d'Alberni), l'Université de Victoria, bien que consciente de la valeur historique inestimable d'une telle collection, a plutôt choisi de retourner les peintures aux artistes (Walsh, 2015). Appuyé par la Commission de vérité et réconciliation du Canada et par des collaborateurs autochtones, l'Université de Victoria a reçu l'approbation d'organiser des expositions des œuvres et de mener des recherches dans le but de retracer les artistes et leurs familles (Walsh, 2015). Ces démarches ont permis de retourner environ 75 pour cent des peintures à leur créateur (Walsh, 2015). Dans tous les cas, des ententes individuelles ont reconnu le droit d'auteur des artistes et ont permis à ceux-ci d'exprimer leur souhait quant à l'utilisation future autorisée de leurs œuvres et à la garde partagée de certains originaux fragiles (Walsh, 2015). Sur ce point, cette initiative de rapatriement se démarque également de la pratique muséale courante par le retour des œuvres à un particulier plutôt qu'à une collectivité. Étant donné la nature délicate des peintures et du contexte traumatisant dans lequel elles ont été réalisées, l'Université de Victoria a choisi de traiter des œuvres comme d'un bien personnel au lieu d'un bien culturel collectif afin de favoriser le processus de guérison des survivants des pensionnats autochtones et de leur famille élargie. Ce choix ne tient peut-être pas compte de la tradition autochtone de propriété intellectuelle collective du savoir traditionnel $^{3}$ - qui inclut, entre autres, la tradition artisanale, la culture matérielle, les connaissances traditionnelles, les rituels, les croyances spirituelles et l'héritage culturel, comme les chants, les histoires et le folklore (Simeone, 2004) ; cependant, la décision de restitution des œuvres aux particuliers semble avoir été prise avec l'accord des collectivités autochtones concernées compte tenu de leur participation au processus d'identification des artistes.

Pour sa part, Bibliothèque et Archives Canada, contrairement aux politiques de rapatriement de la Société du Musée canadien des civilisations, n'a pas entrepris de réformes majeures internes concernant ses relations avec les communautés autochtones. Morse (2012) précise que :

The Library and Archives of Canada Act is completely silent on the place of Aboriginal peoples as consumers of LAC's services, as suppliers and subjects of a sizeable portion of its collection, as co-managers of its materials and as owners of documents originating in their possession (p. 135).

Bibliothèque et Archives Canada (BAC) a toutefois entrepris des démarches en 2003 et en 2005 pour l'établissement d'une stratégie nationale pour le respect des droits des peuples autochtones en matière de propriété intellectuelle, stratégie qui devait être

\footnotetext{
${ }^{3}$ Pour plus d'information sur les questions de droits de propriété intellectuelle autochtone, le lecteur peut consulter IPinCH, 2016 ; Callison, Roy \& LeCheminant, 2016 ; Simeone, 2004.
} 
développée en collaboration avec les communautés et les organisations autochtones (LAC, 2003 ; LAC, 2005).

LAC recognizes the contributions of Aboriginal peoples to the documentary heritage of Canada, and realizes that, in building its collection of these materials, it must take into account the diversity of Aboriginal cultures, the relationship the Government of Canada has with Aboriginal peoples, and the unique needs and realities of Aboriginal communities. The development of a national strategy will be done in consultation and collaboration with Aboriginal communities and organizations, and will respect the ways in which indigenous knowledge and heritage is preserved or ought to be preserved and protected within or outside of Aboriginal communities (LAC, 2005, p. 7).

Ces démarches n'ont toutefois pas abouti à une politique ou à des protocoles nationaux pour les bibliothèques et centres d'archives en fait de rapatriement ou de traitement des documents patrimoniaux autochtones (Morse, 2012, p. 135-136). Les difficultés rencontrées en 2012 par les chercheurs de la Commission de vérité et réconciliation du Canada témoignent également de l'absence de telles politiques. Notons cependant que BAC a tout de même entrepris plusieurs initiatives collaboratives avec les PNMI, entre autres, un cercle de consultation autochtone (2002), un sondage national sur les services autochtones de bibliothèques et de centres d'archives (2008) ainsi qu'une aide financière et professionnelle au Kahnawake Library Project (Cavanagh, 2009, appendice 5, p. 73-79).

\section{Projets de musées, de centres d'archives et de centres culturels autochtones indépendants}

Alors que le rapatriement des restes humains a pour objectif principal le respect des traditions funèbres autochtones et donc le retour ultime de la dépouille à la terre selon la coutume, la restitution des objets sacrés et culturels permet aux peuples autochtones d'entreprendre eux-mêmes des projets culturels par et pour leurs communautés respectives. Cela est d'autant plus important qu'ils dénoncent souvent la représentation en musée de leur patrimoine culturel. C'est le cas, entre autres, des pétroglyphes dorsétiens retirés du site archéologique de Qajartalik au cours des années 1960-70 et exposés pendant un certain temps au Musée canadien de l'histoire (Arsenault \& Gagnon, 2002, p. 145). En plus d'aliéner les lieux sacrés inuits par l'extraction des pétroglyphes, les expositions faites par les conservateurs de l'époque ont défiguré les gravures en rehaussant « la ligne de contour et les traits de la figure rupestre d'une substance pigmentaire rouge [....] Malheureusement, un tel rendu coloré des caractéristiques du pétroglyphe ne s'appuyait sur aucune preuve scientifique » (Arsenault \& Gagnon, 2002, p. 153). De tels exemples soulignent donc l'importance de la préservation in situ de l'héritage culturel autochtone, sinon l'émergence de nouvelles institutions et pratiques muséales typiquement autochtones.

Selon Dubuc et Turgeon (2004), les objectifs autochtones de conservation, de transmission et d'exposition du patrimoine culturel sont souvent animés par une « imagination muséologique » différente de celle de l'institution occidentale (p. 14). 
Tandis que les musées occidentaux ont traditionnellement eu pour mission de rendre compte du passé et donc de traiter des peuples autochtones comme d'une espèce en voie de disparition, les musées et centres culturels autochtones deviennent plutôt des « lieux de vie » et des espaces de création et de valorisation identitaire : « On est loin de l'exposition d'objets inertes pour des étrangers » (Dubuc \& Turgeon, 2004, p. 15.) La culture traditionnelle y est sortie du passé et mise au service de la communauté dans le présent.

Pour sa part, l'Association des archivistes canadiens a publié, en 2007, un guide pour la création de programmes d'archives administrés par et pour les communautés autochtones. L'Aboriginal archives guide (2007) présente les concepts de base de l'archivistique et les étapes à franchir afin de gérer un centre d'archives autochtone : permissions du conseil de bande, choix d'un système de gestion des documents, inventaire des documents, acquisition et rapatriement de documents tenus dans un autre dépôt d'archives, pratiques communes de tenues de documents, calendrier de conservation, plan d'action et budget annuel, entreposage et conservation, demandes de subventions, questions de droit d'auteur, projets d'histoire orale, etc. Par ailleurs, le guide recommande l'embauche d'un archiviste autochtone professionnel, sinon la formation du personnel autochtone. À notre connaissance, ce guide n'a pas donné naissance à un mouvement de construction de centres d'archives au sein des communautés autochtones. Le problème du financement y est sûrement pour quelque chose. Le financement recommandé par la Commission de vérité et réconciliation pour l'examen national des pratiques archivistiques et pour les projets de recherche, de commémoration et de réconciliation donne toutefois espoir de corriger la situation. Par ailleurs, il nous semble probable que le guide a sensibilisé les centres culturels autochtones avec dépôts d'archives à l'importance de la gestion de l'information et aux pratiques archivistiques, notamment en matière d'acquisition, de conservation et de traitement des documents. Cela semble s'avérer pour les nombreux projets d'histoire orale menés de nos jours par plusieurs communautés autochtones soucieuses de conserver la mémoire et les savoir-faire traditionnels de leurs aînés avant qu'il ne soit trop tard (Irving K. Barber Learning Centre, s.d.).

\section{Stratégies de collaboration}

Lorsque le rapatriement ou la création de musées, centres d'archives ou centres culturels autochtones ne sont pas possibles ou souhaitables, faute de ressources matérielles, humaines ou financières, les manifestes nationaux et internationaux recommandent plutôt la création de partenariats et de projets collaboratifs avec les communautés autochtones.

\section{Expositions muséales plurivocales}

Dans les musées, cette approche collaborative, qui passe surtout par l'embauche d'aînés, de conservateurs invités ou d'historiens autochtones, donne lieu à des expositions dites « plurivocales » (Dubuc \& Turgeon, 2004) ou « polyphoniques » (Soulier, 2015) parce qu'elles rendent compte des points de vue multiples : celui du conservateur de musée, souvent allochtone, et ceux des collaborateurs autochtones. 
L'intention étant « de faire ressortir la 'voix authentique' des Amérindiens » dans les expositions muséales (Dubuc \& Turgeon, 2004, p. 11), la plupart des musées d'aujourd'hui adoptent ainsi cette pratique pour l'exposition de leurs collections autochtones.

Dubuc \& Turgeon notent toutefois que l'approche plurivocale :

ne modifie pas fondamentalement les structures du musée, institution qui demeure occidentale dans sa conception, sa visée et son organisation. La mission du musée reste la conservation, la transmission, l'exposition et la mise en valeur du patrimoine dans une perspective essentiellement européenne (2004, p. 13-14).

Si les communautés autochtones se reconnaissent davantage dans ces expositions plus sensibles à leur philosophie, ces dernières demeurent souvent difficiles d'accès pour les PNMI isolées dans leurs communautés et réparties à l'échelle du pays. Ainsi, les expositions des collections autochtones détenues par les grands musées nationaux ou provinciaux, comme le Musée canadien de l'histoire ou encore le Royal Ontario Museum, s'adressent toujours essentiellement à des visiteurs allochtones (Dubuc \& Turgeon, 2004, p. 14).

L'étude de Soulier (2015) vient toutefois confirmer la plus-value des expositions dites plurivocales ou polyphoniques. Dans son analyse d'une exposition collaborative du Musée McCord, Soulier argumente que les visiteurs, tant autochtones qu'allochtones, parviennent à reconnaître et à apprécier la narration multiple de l'exposition. L'auteure souligne que :

Les deux textes d'auteurs [du conservateur du musée et du commissaire autochtone] sont appréciés pour leur complémentarité. En fonction de leurs attentes à l'égard du musée, et aussi de leurs régimes de valeurs et de sensibilités, les visiteurs montrent l'importance et le caractère supplétif des deux visions (Soulier, 2015, p. 111).

Soulier conclut ainsi que la «tyrannie » des points de vue multiples ne semble pas nuire à la capacité communicationnelle des expositions muséales collaboratives (2015, p. 113).

\section{Centres de recherche collaboratifs et projets de numérisation}

La collaboration entre partenaires autochtones, bibliothèques, centres d'archives et centres de recherche donne, pour sa part, lieu à des projets de nature diverse, mais tout aussi intéressants les uns que les autres. Ces projets sont de plus en plus nombreux et la liste d'exemples qui suit est loin d'être exhaustive. Elle donne néanmoins un aperçu des types de projets institutionnels entrepris au cours des dernières années grâce à la collaboration des aînés, des historiens et des chercheurs autochtones. 
Si BAC semble avoir fait peu en matière de rapatriement et de protocoles nationaux d'autonomisation des documents patrimoniaux autochtones comparativement aux musées, l'institution fédérale a tout de même entrepris quelques projets collaboratifs notables. Lancé en 2001 en collaboration avec le collège Nunavut Sivuniksavuk et le gouvernement du Nunavut, le programme Un visage, un nom, est un projet de numérisation, d'étiquetage et de description des collections de photographies autochtones détenues par BAC. D'abord circonscrit à une collection de 500 photographies des communautés inuites, le projet faisait appel aux étudiants du collège Nunavut Sivuniksavuk pour amener les photos numérisées dans leur communauté d'origine et pour communiquer avec les aînés afin d'identifier les lieux, évènements et individus photographiés (BAC, 2005 ; Conseil des académies canadiennes, 2015, p. 85). Depuis, la portée du projet s'est élargie à d'autres collections détenues par BAC et fait appel aux communautés des PNMI d'un peu partout au pays pour l'identification des photographies, et ce, de plus en plus par l'entremise des médias sociaux (blogues, Flickr, Twitter, YouTube) (BAC, 2015).

La création de dépôts numériques et de réseaux de recherche virtuels tels le Great Lakes Research Alliance for the Study of Aboriginal Arts and Culture (GRASAC), le Reciprocal Research Network (RRN), le projet Intellectual Property Issues in Cultural Heritage (IPinCH) et le projet Indigitization du Irving K. Barber Learning Centre, sont également de bons exemples d'initiatives de collaboration.

Créé en 2004 grâce à un partenariat international de chercheurs autochtones, d'universitaires, d'archivistes et de conservateurs de musée, le GRASAC est un centre de recherche virtuel sur le patrimoine culturel des Premières Nations des Grands Lacs dont le siège physique est à l'Université Carleton (GRASAC, 2008). II a aussi pour objectif le rapatriement numérique des collections autochtones de la région, documents qui, au fil des ans, ont été répartis à travers les musées et centres d'archives de l'Amérique du Nord et de l'Europe (GRASAC, 2008).

Le RRN a, pour sa part, été fondé par le Musée d'anthropologie de l'Université de la Colombie-Britannique en collaboration avec la bande Musqueam, le conseil tribal de la nation Stó:Iō et la société culturelle U'mista. II s'agit d'un réseau de chercheurs autochtones et allochtones se ralliant autour d'un dépôt numérique constitué des collections de 24 institutions muséales et archivistiques de la côte nord-ouest britannocolombienne (RRN, 2014).

Mené par l'Université Simon Fraser, IPinCH est un projet de recherche interdisciplinaire et international qui s'intéresse aux questions théoriques, éthiques, politiques et légales de l'héritage culturel et des droits de propriété intellectuelle autochtones. Le projet rassemble plus de 50 chercheurs, 25 organismes et 12 nations autochtones venant du Canada, de l'Australie, des États-Unis de la Nouvelle-Zélande, de l'Afrique du Sud, de l'Allemagne, de l'Angleterre et de la Suisse (IPinCH, 2016).

Par ailleurs, Indigitization, initiative du Irving K. Barber Learning Centre, du First Nations Technology Council et des communautés autochtones Heiltsuk, Ktunaxa et 'Namgis, est un projet de numérisation des savoirs traditionnels autochtones. Le projet offre des 
services de numérisation d'enregistrements de projets d'histoire orale autochtones ainsi qu'un coffre à outils pour la numérisation, la gestion de données et la formation du personnel archivistique autochtone (Irving K. Barber Learning Centre, s.d.).

\section{Réflexions et recommandations}

L'analyse comparative précédente des stratégies d'autonomisation et de collaboration nous permet d'avancer quelques réflexions et recommandations en matière de décolonisation de la recherche et de la mémoire autochtones.

D’abord, il nous paraît que les stratégies d'autonomisation décrites dans cet article sont celles les plus alignées sur les recommandations de la Déclaration des Nations Unies sur les droits des peuples autochtones, de la politique sur les données autochtones PCAP et de la Commission de vérité et réconciliation du Canada. En redonnant aux communautés autochtones la propriété et le contrôle de leurs restes humains et de leurs objets sacrés et culturels, les politiques de rapatriement des institutions de la mémoire et du savoir reconnaissent officiellement les injustices de la colonisation et cherchent activement la réparation des torts par la réconciliation avec les PNMI. Reste que l'absence d'un cadre législatif national en matière de rapatriement fait en sorte qu'il existe des différences notables entre pratiques institutionnelles à l'échelle du pays. Par ailleurs, il incombe toujours aux PNMI la responsabilité de revendiquer officiellement la propriété et le retour de leur patrimoine culturel et de faire la démonstration de leur lien historique aux objets revendiqués. Le rapatriement imposé d'office d'une collection de peintures autochtones détenues par l'Université de Victoria fait toutefois figure d'exception. En assumant la responsabilité de recherche d'identification des artistes et l'initiative du retour, I'Université de Victoria s'impose, à notre avis, comme modèle de réconciliation et exemple à suivre, et ce, malgré le fait qu'une telle démarche exige que l'institution contrevienne au principe fondamental du respect du fonds en archivistique.

Parallèlement, en cédant une partie de leurs collections aux PNMI, les institutions de la mémoire facilitent la création de musées, de centres d'archives ou de centres culturels gérés par et pour les communautés autochtones. Ces initiatives permettent aux peuples autochtones de reconquérir leur passé et de proposer une interprétation de leur histoire et de leurs traditions qui tient davantage compte de leurs réalités et philosophies. Ces représentations du passé répondent souvent à un mandat présentiste, c'est-à-dire au besoin de renouer avec la culture ancestrale et d'assurer la vitalité culturelle en créant des « lieux de vie » où le passé est au service du présent. Conformément au droit des peuples autochtones à l'autodétermination, l'autonomisation et l'autogestion des musées, centres d'archives et centres culturels autochtones permettent donc aux PNMI de se réconcilier avec l'histoire coloniale et de reprendre en main leur passé de même que leur avenir. Pour atteindre ces objectifs, par contre, le gouvernement canadien devra toutefois contribuer au financement de plusieurs de ces projets qui sont loin d'avoir les budgets et les ressources humaines et matérielles des institutions fédérales comme le Musée canadien de l'histoire ou encore BAC. Ces ressources financières nous semblent nécessaires au maintien des conditions minimales de préservation, de traitement et de transmission des précieuses collections cédées aux communautés autochtones. À cet égard, donc, nos recommandations rejoignent celles de la 
Commission de vérité et réconciliation quant au financement fédéral de projets de recherche, de commémoration et de réconciliation.

D'autre part, les stratégies de collaboration entre communautés autochtones et les institutions occidentales de la mémoire s'inscrivent plutôt dans la logique des recommandations du Groupe de travail sur les musées et les PNMI de 1992. S'il faisait la promotion du rapatriement dans certaines circonstances, le Groupe de travail reconnaissait tout de même l'expertise et l'important travail des musées en matière de préservation et de sensibilisation du public au patrimoine culturel des peuples autochtones du pays (AMC \& APN, 1992, p. 6). Ainsi, dans un esprit de respect mutuel, il recommandait surtout l'accès amélioré des PNMI aux collections autochtones, aux cadres administratifs et aux montages des expositions des musées. Ces recommandations semblent effectivement avoir donné lieu à des expositions muséales plus nuancées et plus fidèles à l'expérience autochtone, cependant elles demeurent, la plupart du temps, physiquement très éloignées des PNMI représentées et profitent surtout à un public non autochtone. De telles expositions servent alors à sensibiliser et à enrichir les connaissances des visiteurs allochtones quant à la richesse culturelle des PNMI, sans nécessairement contribuer à la vitalité culturelle et encore moins au développement économique et touristique des collectivités autochtones visées. Des projets d'expositions virtuelles collaboratives pourraient cependant remédier, en partie, à cette situation par le rapatriement numérique.

C'est d'ailleurs ce qui semble faire la force des projets collaboratifs de recherche et de numérisation, tels ceux du GRASAC, du RRN et du projet Indigitization. Par leurs partenariats avec les chercheurs et les communautés autochtones, ces projets rendent davantage service aux PNMI en leur donnant plus facilement accès à leur patrimoine documentaire et culturel, et ce, par le rapatriement numérique et la numérisation de projets d'histoire orale. Alors que leurs objectifs principaux varient légèrement, ces initiatives sont néanmoins généralement animées par un esprit d'entraide mutuel, profitant de l'expertise, des savoirs et des connaissances multidisciplinaires des membres - tant autochtones qu'allochtones - pour le bien des études autochtones. Ainsi, il y a généralement un effort de formation du personnel autochtone, notamment en matière de numérisation et de pratiques archivistiques. Il est toutefois à noter que le patrimoine documentaire et culturel amassé et préservé par ces centres profite surtout à leurs membres et aux partenaires autochtones, étant donné l'existence de certaines restrictions d'accès liées à la propriété intellectuelle, aux renseignements privés et confidentiels et aux croyances spirituelles autochtones à l'égard de certains objets ou documents sacrés (GRASAC, 2008). Le libre accès à ces réseaux et ressources autochtones est donc loin d'être une réalité.

En somme, nos recommandations vont dans le même sens que celles formulées en 1992 par le Groupe de travail sur les musées et les PNMI. II nous paraît essentiel de continuer à sensibiliser le personnel des musées, centres d'archives et bibliothèques à l'histoire et aux enjeux du mouvement pour la réappropriation du patrimoine culturel autochtone. Suivant les recommandations de la Commission de vérité et réconciliation du Canada, les institutions seront d'ailleurs appelées à rendre davantage compte de l'histoire des pensionnats autochtones (2015, art. 68-69, p. 8). Des formations 
obligatoires rappelant l'histoire coloniale, le contexte de décolonisation et de revendications autochtones ainsi que les droits autochtones en matière d'héritage culturel devraient ainsi guider le personnel de ces institutions à mieux choisir la stratégie (d'autonomisation ou de collaboration) à adopter dans la mise en application de ces recommandations.

À cet égard, nous croyons que les initiatives adoptées par les musées, centres d'archives ou bibliothèques - qu'elles soient des stratégies d'autonomisation ou de collaboration - doivent se faire en consultation avec les organismes et les communautés autochtones concernés. Étant donné le caractère distinct et les circonstances variées de chaque Première Nation, il revient à chacune d'elles de déterminer des mesures acceptables et applicables pour leurs communautés. II sera donc important pour les institutions occidentales de la mémoire détenant des collections autochtones de continuer à développer et à nourrir leurs partenariats avec les PNMI.

Si dans le passé les efforts de préservation des institutions de la mémoire et des collectivités autochtones se sont surtout concentrés sur la mémoire des aînés, la culture et les savoir-faire traditionnels, il ne faudrait toutefois pas perdre de vue les documents et contenus autochtones créés numériquement dès l'origine, notamment par les nouvelles générations qui s'expriment de plus en plus par l'entremise des médias sociaux numériques. Le Conseil des académies canadiennes prévient d'ailleurs les institutions de la mémoire contre la création d'un « trou noir numérique dans les documents historiques des nations » faute d'un archivage immédiat du patrimoine culturel créé numériquement (Conseil des académies canadiennes, 2015, p 30). Ainsi, les institutions de la mémoire et les collectivités autochtones devraient établir des procédures pour l'acquisition et la préservation de ces témoignages de la jeunesse autochtone afin de prévenir qu'ils se perdent dans la surabondance de l'information du Web.

En résumé, nous recommandons aux institutions canadiennes de la mémoire de :

- continuer à sensibiliser leur personnel aux enjeux de la réappropriation du patrimoine culturel autochtone ;

- continuer à développer et à nourrir leurs partenariats avec les PNMI ;

- favoriser, dans la mesure du possible, les stratégies d'autonomisation, c'est-àdire le rapatriement des biens culturels autochtones et la création d'institutions de la mémoire typiquement autochtones ;

- favoriser des pratiques de rapatriement d'office plutôt que sur demande officielle des communautés autochtones ;

- veiller à la préservation du patrimoine culturel autochtone créé numériquement afin de conserver la mémoire des nouvelles générations qui s'expriment de plus en plus par l'entremise des médias sociaux; 
- exploiter plus à fond le potentiel des technologies numériques pour la réappropriation du patrimoine culturel et documentaire autochtone : rapatriement numérique, expositions virtuelles, collections numériques, utilisation des réseaux et des médias sociaux, etc.

De plus, nous recommandons au gouvernement fédéral de :

- élaborer un cadre législatif national en matière de rapatriement afin de normaliser les pratiques institutionnelles ;

- assurer un financement adéquat aux programmes d'institutions de la mémoire autochtones afin d'assurer leurs capacités de maintenir les conditions minimales de préservation, de traitement et de transmission de leurs collections.

\section{Bibliographie}

Arsenault, D. \& Gagnon, L. (2002). Les représentations rupestres autochtones : du lieu in situ au lieu muséal : perte ou enrichissement ? Ethnologies, 24(2), 139-160. DOI : 10.7202/006643ar.

Assemblée des Premières Nations. (2007). PCAP : Propriété, contrôle, accès et possession. Données sur le droit inhérent des Premières Nations à régir leurs données. Ottawa : Assemblée des Premières Nations.

Association des femmes autochtones du Canada. (2011). Un protocole d'application culturellement pertinent selon les sexes. Ottawa : Association des femmes autochtones du Canada.

Association des musées canadiens / Assemblée des Premières Nations. (1992). Tourner la page : forger de nouveaux partenariats entre les musées et les Premières Nations. Rapport du groupe de travail sur les musées et les Premières nations. Ottawa : Groupe de travail sur les musées et les Premières Nations.

Association des Premières Nations du Québec et du Labrador. (2014). Protocole de recherche des Premières Nations au Québec et au Labrador. Wendake : Assemblée des Premières Nations du Québec et du Labrador.

Association of Canadian Archivists Public Awareness Committee. (2007). Aboriginal archives guide. Canadian Church Historical Society Occasional Paper, 8. Récupéré le 13 juin 2015.

Bibliothèque et Archives Canada. (2009). Un visage, un nom. Récupéré le 13 juin 2015. 
Bibliothèque et Archives Canada. (2015). Un visage, un nom. Récupéré le 13 juin 2015.

Callison, C., Roy, L. \& LeCheminant, G. (2016). Indigenous notions of ownership and libraries, archives and museums. Berlin/Munich : De Gruyter Saur.

Cavanagh, M. (2009). Sound practices in library services to Aboriginal Peoples: Integrating relationships, resources and realities. Aboriginal Library Services Working Group, Provincial/Territorial Public Library Council. Récupéré le 16 juin 2015.

Conseil des académies canadiennes. (2015). À la fine pointe du monde numérique : possibilités pour les institutions de la mémoire collective au Canada. Ottawa, ON : Le comité d'experts sur les institutions de la mémoire collective et la révolution numérique, Conseil des académies canadiennes. Récupéré le 6 juin 2015.

Dickason, O. P. (2006). Tightening the reins: Resistance grows and organizes. Dans A concise history of Canada's First Nations (p. 225-241). New York : Oxford University Press.

Dubuc, E. \& Turgeon, L. (2004). Musées et Premières Nations : la trace du passé, l'empreinte du futur. Anthropologie et Sociétés, 28(2), 7-18.

Edwards, S. (2007, 14 septembre). Native peoples declaration 'unworkable'; Canada among four nations to oppose UN stand. Edmonton Journal, p. A4.

Femmes autochtones du Québec Inc. (2012). Lignes directrices en matière de recherche avec les femmes autochtones. Kahnawake: Femmes autochtones du Québec Inc.

Gouvernement du Canada. (1982). Loi de 1982 sur le Canada: Charte canadienne des droits et libertés. L.C. c. 11, art. 25. Récupéré le 27 mai 2015.

Gouvernement du Canada. (2015, 2 juin). Débats de la Chambre des communes. Hansard révisé, 147(222). 41e législature, $2^{\mathrm{e}}$ session. Récupéré le 16 juin 2015.

Government of Alberta. $(2000,2008)$. First Nations Sacred Ceremonial Objects Repatriation Act. RSA 2000, C F-14. Récupéré le 30 mai 2015.

Government of Alberta. (2004, 2014). Blackfoot First Nations Sacred Ceremonial Objects Repatriation Regulation. AR 96/2004, AR 59/2014. Récupéré le 30 mai 2015.

Government of Canada (1999). Nisga'a Final Agreement. Récupéré le 28 mai 2015.

Government of the United States. (1990). Native American Graves Protection and Repatriation Act. 25 U.S.C. 3001 et seq. Récupéré le 9 avril 2015. 
Great Lakes Research Alliance for the Study of Aboriginal Arts and Culture. (2008). About GRASAC. Récupéré le $1^{\mathrm{er}}$ juin 2015.

IPinCH. (2016). Intellectual property issues in cultural heritage: Theory, practice, policy, ethics. Récupéré le 3 octobre 2016.

Irving K. Barber Learning Centre. (s. d.). Indigitization: Toolkit for the digitization of First Nations knowledge. Récupéré le 6 avril 2015.

Lambert, S. (2007, 14 septembre). Canada under fire for vote against UN native-rights policy. The Globe and Mail, A17.

Library and Archives Canada. (2003). Report and recommendations of the consultation on aboriginal resources and services. Récupéré le 31 mai 2015.

Library and Archives Canada. (2005). Collection development framework. Récupéré le 31 mai 2015.

Lum, Z.-A. (2014, 3 octobre). Canada is the only UN member to reject landmark indigenous rights document. Huffington Post. Récupéré le 18 mai 2015.

Morse, B.W. (2012). Indigenous human rights and knowledge in archives, museums, and libraries: Some international perspectives with specific reference to New Zealand and Canada. Archival Science, 12(2), 113-140.

Nations Unies. (1948). Déclaration universelle des droits de l'homme. Récupéré le 16 juin 2015.

Nations Unies. (2007). Déclaration des Nations Unies sur les droits des peuples autochtones. Récupéré le 9 avril 2015.

Parti libéral du Canada. (2015). Vérité et réconciliation. Récupéré le 2 décembre 2015.

Reciprocal Research Network. (2014). About the RRN. Récupéré le 20 mai 2015.

Royal British Columbia Museum. $(2003,2012)$. Aboriginal material operating policy. Récupéré le 30 mai 2015.

Royal Ontario Museum. (2001, 2012). Board policy: Repatriation of Canadian Aboriginal objects. Récupéré le 30 mai 2015.

Royal Ontario Museum. (2002, 2012). Board policy: Repatriation of human remains of the Aboriginal Peoples of Canada. Récupéré le 30 mai 2015.

Simeone, T. (2004). Connaissances traditionnelles autochtones et droits de propriété intellectuelle (PRB 03-38F). Ottawa : Division des affaires politiques et sociales. Récupéré le 3 octobre 2016. 
Société du Musée canadien des civilisations. (2001). Politique sur le rapatriement. Récupéré le 30 mai 2015.

Soulier, V. (2015). Analyser la reconnaissance du point de vue autochtone dans une exposition muséale. Éducation et francophonie, 43(1), 97-115.

Truth and Reconciliation Commission of Canada. (2012). Truth and Reconciliation Commission of Canada: Interim report. Winnipeg: Truth and Reconciliation Commission of Canada. Récupéré le 9 avril 2015.

Truth and Reconciliation Commission of Canada. (2015). Truth and Reconciliation Commission of Canada: Calls to action. Winnipeg: Truth and Reconciliation Commission of Canada. Récupéré le 3 juin 2015.

Truth and Reconciliation Commission of Canada. (s.d.). Honouring the truth, reconciling for the future: Summary of the final report of the Truth and Reconciliation Commission of Canada. Récupéré le 16 juin 2015.

Tuhiwai Smith, L. (2008). Decolonizing Methodologies: Research and Indigenous Peoples. Dunedin : University of Otago Press.

United Nations. (2014, 15 septembre). Outcome document to be adopted by the General Assembly on 22 September 2014. Récupéré le 20 mai 2015.

Walsh, A. N. (2015, 1 juin). Childhood paintings from the Alberni Indian Residential School: A story of return and reconciliation. Présentation donnée dans le cadre des activités de clôture de la Commission de vérité et réconciliation du Canada, Gatineau : Musée canadien de l'histoire. 